\title{
Virtual Reality Systems and Applications: The Ancient Olympic Games
}

\author{
A. Gaitatzes, D. Christopoulos, and G. Papaioannou \\ Foundation of the Hellenic World, 38 Poulopoulou St., \\ 11851 Athens, Greece \\ \{gaitat, christop, gepap\}@fhw.gr
}

\begin{abstract}
This paper presents the virtual reality systems, interaction devices and software used at the Foundation of the Hellenic World (FHW). The applications that FHW has produced, associated with the Olympic Games in ancient Greece, are then detailed. The separate virtual reality shows are presented in terms of interactivity and educational value. Technical aspects of the productions are explained, with an emphasis on surround screen projection environments. These techniques were mostly utilized in the recent production regarding the ancient Olympic Games, where much effort has been made to recreate the feeling of the games and help the user/spectator be an interacting part of the edutainment activity.
\end{abstract}

\section{Introduction}

The undertaking of the $28^{\text {th }}$ Summer Olympiad by Athens, the capital city of Greece, has spurred an increased interest for shows and complementary edutainment contributions to the games themselves. The Foundation of the Hellenic World (FHW) has prepared a series of thematic productions related to ancient Olympia and the Olympic Games, culminating with the highly interactive and accurate representation of ancient Olympia and the pentathlon (running, long jump, javelin, discus throwing and wrestling), whose final version was released just before the beginning of the Olympic Games. The high demand for interactive and entertaining productions, apart from simple walkthrough applications, however eye-catching, has driven us to pursue an interaction model different from the classic navigator/inspector one. The virtual reality (VR) productions should be educational through creativity and active participation in events [9]. Our audience has confirmed their preference toward more "playable" environments where experimentation and first-hand experience is the most important channel leading to knowledge.

In the rest of the paper, section 2 provides the linking background and a brief insight to each one of the thematic applications, while section 3 explains the key points of the scientific and technological features related to the interactive shows and our effort to implement these in a surround screen projection environment.

\section{The Productions}

In 2000, as the Olympic Games were returning to their birthplace, we decided to focus the new projects on the thematic region of the Olympic History and Games for the 
virtual reality productions that would open in the time period before the Olympic Games in Athens. Instead of focusing on only one production, where every concept and aspect of this glorious event would be explained and presented, several productions were created, each one complementary to the previous and finally concluding in a full-scale interactive representation of ancient Olympia and its Games.

\subsection{Olympic Pottery Puzzle}

The study of ancient pottery has been a very important source of historical and archaeological information for understanding the life and culture in ancient Greece. Apart from the functional significance of the various types of pots, which give us clues about rituals and everyday life, the ink-paintings that cover many pot shards found at excavation sites, are priceless since they allow us to have an inside look into history. The goal of the Olympic pottery puzzle application was to educate the visitors about all this information, emphasizing on Olympic Athletic events in an entertaining and understandable way, using the constructivist's approach [13] (Fig. 1).

The user must re-assemble a number of ancient vases putting together pot shards. The users are presented with a color-coded skeleton of the vessels with the different colors showing the correct position of the pieces. They then try to select one piece at a time from a heap and place it in the correct position on the vase. When they finish the puzzle, the painting on the vase comes to life, presenting an animation of one of the ancient Olympic contests. From a technical perspective, much effort was directed towards the realistic, highly detailed representation of vases and the simulation of their material properties like specularity and glossiness, using multi-pass rendering techniques.

This VR exhibit captivates the visitors, giving them the opportunity to interact with the vases intuitively. Completing the $3 \mathrm{D}$ assemblage puzzle with the help of VR equipment helps them learn some important historical facts about the vases and the Olympic contests, as well as have a glimpse of the restoration procedure of earthenware. The reanimation of the depicted athletes was made in $2 \mathrm{D}$ (planar video over-
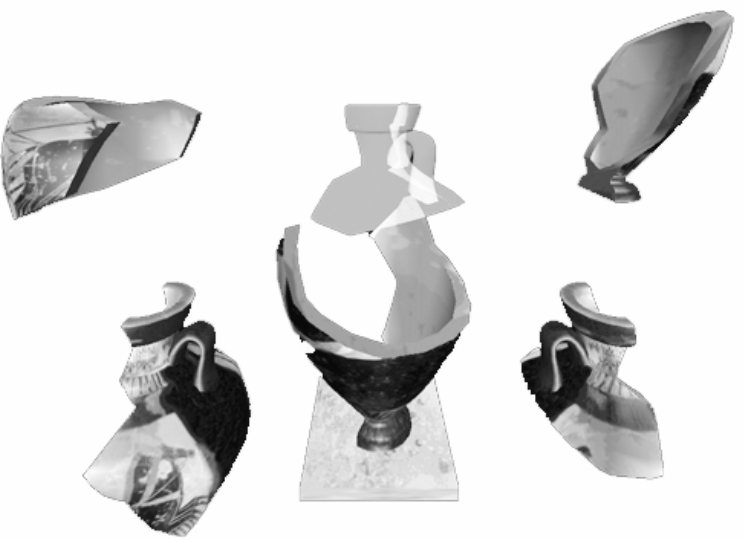

Fig. 1. The Olympic pottery puzzle 
lays), preserving the colors and character of the ancient Greek ink paintings. This, in turn, helped the visitors perceive the Olympic contests and any information regarding the Olympic Games in antiquity from the perspective of an ancient Greek artist, something the public responded enthusiastically to. The "Olympic Pottery Puzzle" is suitable for presentation in both single-screen and surround screen projection systems.

\subsection{Feidias' Workshop}

After the successful launch of the first interactive application regarding the Olympics, it was important to build on the positive feedback and winning concepts and create an experience, which would bring the visitor one step closer to Olympia. In this VR exhibit, visitors engage themselves in another marvel of ancient Greek art, the creation of golden ivory statues. Golden ivory statues are regarded as the masterpieces of Greek sculpture, and were admired as such even at the time of their creation. Only statues of Gods and heroes were made using this technique.

"Feidias' Workshop" (Fig. 2) is a highly interactive virtual experience that takes place at the construction site of the 15-meter-tall golden ivory statue of Zeus. The visitors enter the two-story-high workshop and come in sight of an accurate reconstruction of an unfinished version of the famous statue of Zeus and walk among the sculptor's tools, scaffolding, benches, materials, and moulds used to construct it. The visitors take the role of the sculptor's assistants and actively help finish the creation of the huge statue, by using virtual tools to apply the necessary materials onto the statue, process the ivory and gold plates, apply them onto the wooden supporting core and add the finishing touches. Interaction is achieved using the navigation wand of the VR system [9], onto which the various virtual tools are attached. Using these tools the user helps finish the work on the statue, learning about the procedures, materials and techniques applied to the creation of these marvelous statues. The various workers and Feidias himself are displayed using image-based rendering techniques in the form

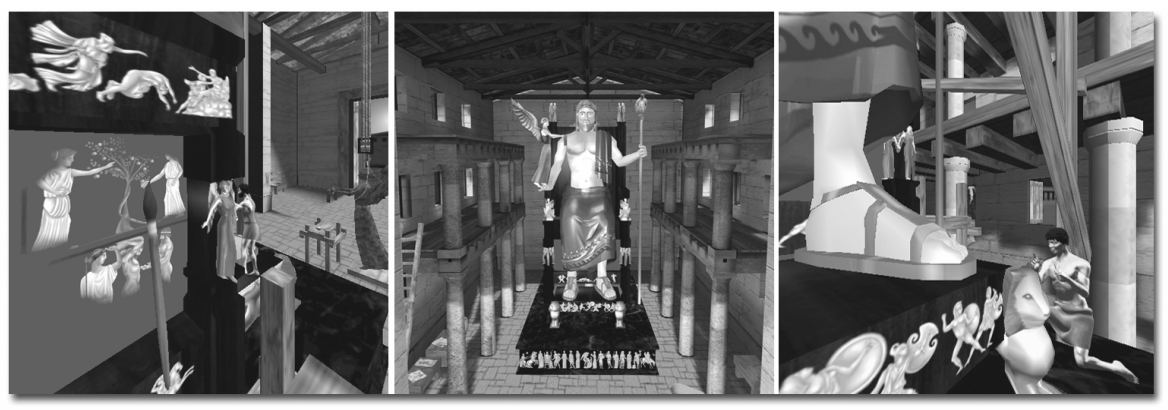

Fig. 2. A creative approach to interactive edutainment

of animated impostors [11]. This technique was chosen because of its low polygon count since the detail of the other models in the scene was high. In order to provide additional depth cues for the interaction and better image realism, pre-computed illumination was also applied using lightmaps. 
This VR production presents an accurate reconstruction of a populated and active workshop, with interactive and educational value. The "learning by doing" concept worked well and increased the visitors' interest and active participation in the VR show. The use of the CAVE-like environment helps support the large scale and proportions of the workshop and the statue of Zeus. The positive feedback we got from the incorporation of characters, led us to the conclusion that the public was especially interested in the way of life and working habits of people in ancient Greece. The use of animated representation of characters helped the audience understand, participate and immerse themselves into the experience, giving them a reference point of interest.

\subsection{A Walk-Through Ancient Olympia}

In our most recent VR production "A Walk through Ancient Olympia”, the user visiting the virtual historical site, learns about the ancient games themselves by interacting with athletes in the ancient game of pentathlon (Fig. 3).

We are at the end of the 2 nd century BC. The day breaks and in front of us appears the majestic sanctuary of Zeus in ancient Olympia. In antiquity the Olympic Games took place here, while today it hosts the lighting of the Olympic flame. The visitors can wonder around and visit the buildings and learn their history and their function.

In addition, the public can interact virtually with 3D digital representations of Olympian athletes in the ancient pentathlon, including the 200-meter sprint, the discus throwing, the long jump, the javelin throwing and wrestling. Instead of just observing the games the visitors take part in them. They pick up the discus or the javelin and they try their abilities in throwing them towards the far end of the stadium. A roleplaying model of interaction with alternating roles was tried here with pretty good success as the visitors truly immersed in the environment wish they could participate in more games.

Finally, decorated with red ribbons in his hands and legs, the glorious winner makes the tour of triumph holding a palm leaf in his hand while the spectators give him a standing ovation. The Games come to an end.

\section{Production Technology}

From a technical point of view, the productions of FHW are presented on two VR systems, both using projective viewing technology [8]. The larger system is a CAVElike ReaCTor ${ }^{\mathrm{TM}}$ immersive display [5], consisting of four $3 \mathrm{~m} \times 3 \mathrm{~m}$ projection screens. An 8-processor SGI ${ }^{\circledR}$ Onyx $2^{\mathrm{TM}}$ drives the four projectors through four InfiniteReality $2^{\mathrm{TM}}$ graphics subsystems, in active stereo. A maximum number of 10 visitors and a museum educator enter the immersive cube, all wearing stereo shutter glasses and the show is controlled by the guide via a six degrees-of-freedom tracked joystick. The museum educator also wares a hat with an attached six degrees-of-freedom sensor for the head position and orientation tracking, which affects the perspective projection on each wall. 


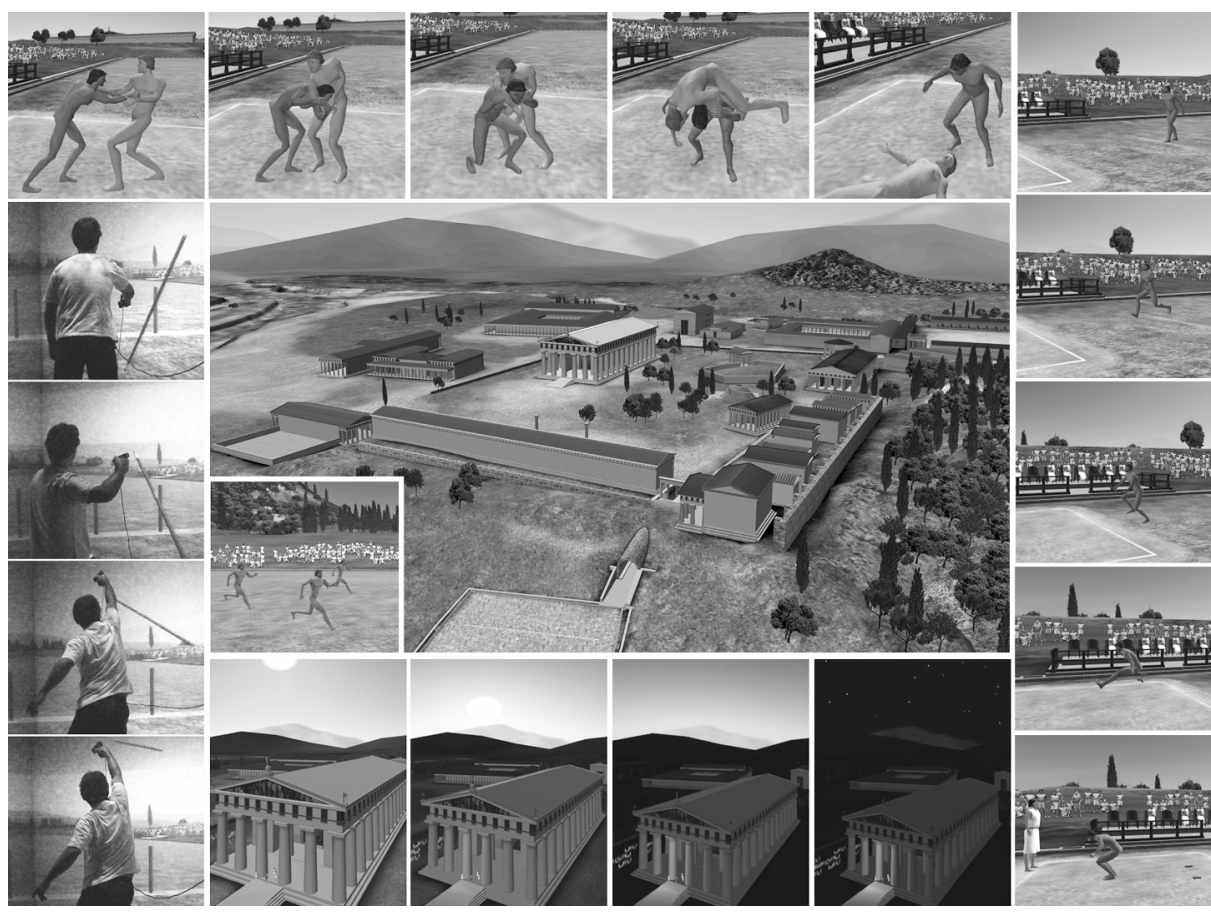

Fig. 3. Screenshots and live capture from the production "A Walk through Ancient Olympia"

The smaller VR system consists of an ImmersaDesk ${ }^{\mathrm{TM}} \mathrm{R} 2$ rear projection tilted screen driven in active stereo by an $\mathrm{SGI}^{\circledR}$ Octane $2^{\mathrm{TM}}$. Six degrees-of-freedom head and hand tracking are provided via a head-mounted tracker and a tracked joystick. This system, having a lesser immersive effect and also a much slower computing system, is used mostly for lightweight applications. The large interactive VR shows are run in the surround-screen projection environment.

In terms of software technology, we have built our own game engine, Enhanced Visualization System (EVS) [11], [12], [8], using C++, based on OpenGL Performer $^{\mathrm{TM}}\left[15\right.$ and OpenGL $^{\circledR}$ for the graphics, on the CAVElib and VRPN [16] libraries for transparent access to the virtual reality hardware and stereoscopic rendering and a customary developed sound library for playing audio.

The system is divided into two major components: the scripting language, which describes the scene as a collection of nodes and their connection via events and messages and the low level core $\mathrm{C}++$ classes that implement the features and interpret the scripting language commands. Thus the authors have to mostly create scene files (ascii text), where a description of the world using the scripting language is stored. The framework includes many of the features common to virtual environments and allows engineers to reuse tools and code between various applications and at the same time incorporate new features. Artists can participate more actively or even develop entire applications on their own, adjusting the final virtual environment to their needs. The framework allows for multithreaded execution, which is essential for interaction in a 

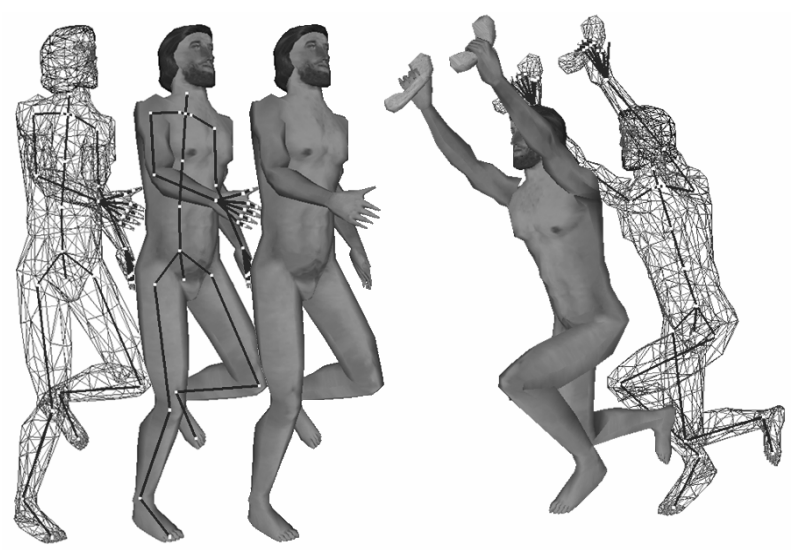

Fig. 4. Character animation using bones and soft skinning

multiprocessor system such as the ones used normally in VR where each projection surface has its own Graphics/Raster engine.

For navigation and interaction a wide range of devices are supported. The VRPN [16] integration into EVS enables the usage of low-cost devices such as mice and joysticks and the usage of high-end tracker solutions such as the Intersence IS-900 using a common interface.

As natural interaction requires the presence of familiar and realistic representations not just of visual aspects of the virtual world, but also of actions and procedures, important techniques and features such as character animation and dynamics have been incorporated into the VR engine and are discussed below.

\subsection{Character Animation}

Real-time character animation in simulated virtual environments has progressed rapidly over the last years. This development has also been reflected by the changes of the visual style in virtual environment applications. When the experience is to be enriched with interaction in active environments where people should explore and participate in close interaction with virtual people, the use of 3D animated characters, with recognizable and natural features, gestures and fluid motion, is essential.

The animation technique, which has established itself as the defacto way in bringing articulated models to life in real-time 3D environments, is skeletal animation combined with soft skinning.

Skeletal animation uses an endoskeleton, a hierarchic structure of joints and bones, which drives a skin, a vertex mesh representing the outer shell of the object (Fig. 4). All the bones of the articulated object form together the skeleton. Only the skeleton is explicitly animated, which in turn implicitly animates the skin and its vertices. Using soft skinning, each vertex of the mesh can be influenced by more than one bones [18], effectively mimicking the way a bone in a real body would affect the skin of a living being. Memory usage for skeletal animation is small and requires a significantly lower amount of information to be stored when compared to other techniques. Anima- 
tion data can also be generated on the fly using techniques like inverse kinematics [17], and applied to the model in real-time.

For our recent VR production "A Walk through Ancient Olympia" the skeletal animation technique was implemented for animating the various characters and athletes (Fig. 4). Using the Cal3D animation framework library [1], specialized EVS rendering and simulation nodes were created which allowed us to incorporate skeletal animations from commercial modeling and animation software into our framework. Besides the playback of animations, the ability to blend and mix multiple animations was implemented for advanced animation control, generating smooth transitions between the animations and dispensing with extra transition animation cues.

Due to the fact that models also have to interact with the environment by picking and dropping other objects, a mechanism for attachment and detachment of other scene objects on the characters was implemented.

\subsection{Newtonian Dynamics}

One of the challenges in the "Walk through Ancient Olympia" was to be able to actively participate in at least one of the games (Fig. 3). Considering the difficulty to move in a CAVE when surrounded by spectators, we decided to implement such interaction for the discus and javelin throwing games, which are relatively static. Both games required that the user take hold of an object and send it flying through the scene under the influence of forces in a controllable manner. The object would collide, bounce off and exchange energy with other geometry. For this purpose we used Newtonian dynamics, a well-known and extensively studied motion model for rigid bodies and their response to collisions [3], [6].

\subsection{Occlusion Culling for Open Environments}

As graphics cards have a limit to the fill rate and triangle count the application can pump into them, culling - or non-visible geometry elimination - is used extensively in large 3D worlds in order to minimize the geometry sent for rendering in the graphics card. In occlusion culling [2], geometry that is hidden behind objects closer to the camera point, is discarded before being subject to depth sorting algorithms. Shadow culling [10], uses predefined occlusion proxies (occluders), which consist of simplified (and usually convex) polygonal versions of actual rendered geometry, such as rectangular barriers, in order to efficiently block geometry hidden behind them. Shadow culling is best suited for our outdoor sparse environments as compared to other techniques, most of which are targeted for dense or indoor scenes.

In each frame, a semi-infinite convex frustum is created for each (convex) occluder polygon, the cap of the semi-infinite frustum being the occluder polygon itself and the sides connecting the each edge with the viewpoint (Fig. 5). The bounding boxes of the geometry to be rendered are compared for containment with these frusta. If a bounding box resides completely within all frusta, then the object it contains is hidden. This process is performed in a hierarchical manner, discarding whole sub-trees of a 3D scene graph prior to forwarding the geometry to the rendering engine. Careful construction and placement of the occluders [7], results in high hidden geometry elimination and a considerable speedup. 

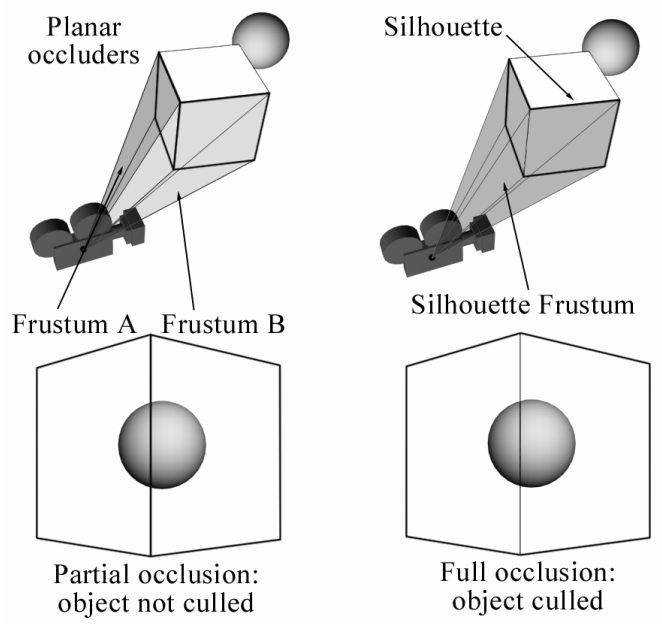

Fig. 5. Comparison between polygonal and solid occluders in shadow culling

\subsubsection{Solid Occluders}

In the case of outdoor scenes, like Olympia, where the static geometry is often blocky (buildings) but scattered and sparse, simple occlusion culling is not very effective due to the partial occlusion phenomenon (Fig. 5): Two or more adjoining planar occluders may partially hide a distant object but the combined occlusion area of them may hide it completely. This object cannot be eliminated if the frustum for each occluder is created separately, and the joining of frusta is an expensive operation.

To overcome this taxing limitation, we decided to use convex solid occluders [7], such as boxes and cylinders as proxies for large isolated structures (Fig. 5). A convex solid when projected on a plane is guaranteed to produce a convex polygon. The convex frustum of the projected polygon is the union of the frusta that would be generated from the individual planes of the solid occluder, thus bypassing the need to merge frusta in order to avoid partial occlusion.

For each frame, the solid occluder frustum is generated as follows. The view dependent silhouette of the solid occluder is extracted by connecting the edges belonging to adjacent polygons, which are not both visible or hidden simultaneously:

$$
\begin{aligned}
& \text { edge }\left(t_{i}, t r_{j}\right) \in \text { Silhouette } \Leftrightarrow \\
& {\left[\vec{N}_{i} \cdot\left(\bar{P}_{i 0}-\bar{C}\right)\right] \cdot\left[\vec{N}_{j} \cdot\left(\bar{P}_{j 0}-\bar{C}\right)\right] \leq 0}
\end{aligned}
$$

where $\vec{N}_{k}$ is the normal vector of triangle $t r_{k}, \bar{P}_{k 0}$ the triangle's first point and $\bar{C}$ the viewpoint.

The Silhouette edges do not lie on the same plane in general. Therefore, we must select a cap (near plane) for the semi-infinite frustum, based on the relative position of the viewer and the silhouette points. We chose to fix the near plane of the frustum to the furthest point of the silhouette from the viewer in order to avoid false positive 
culling results. This clipping plane's normal vector is the average directional vector between the viewpoint and the silhouette points $\bar{S}_{i}$ :

$$
\vec{N}_{\text {near }}=\sum_{i=0}^{K-1}\left(\bar{S}_{i}-\bar{C}\right) /\left\|\sum_{i=0}^{K-1}\left(\bar{S}_{i}-\bar{C}\right)\right\|
$$

\subsubsection{Occluder Selection}

As Hudson et al. [10] suggest, a scene may contain too many occluders for the engine to be able to test each object against each one of them. In our case, Olympia contains more than 200 occluder planes and solids. Therefore, an optimal set of occluders has to be selected for each frame at run-time in order to keep the number of "active" occluder primitives to a minimum. For this task, a "score" or optimization function $f_{\text {planar }}$ has to be devised that takes into account the solid angle of the frustum. Hudson et al. use the area-angle approximation presented by Coorg and Teller [4]:

$$
f_{\text {planar }}=\frac{-A \vec{N} \cdot \vec{V}}{\|\vec{V}\|^{2}}
$$

where $A$ is the area of a planar occluder, $\vec{N}$ is its normal vector and $\vec{V}$ is the vector from the viewpoint to the centre of the occluder. We use the criterion in eq. (3) for planar occluders. For solid occluders we use an approximation formula that depends on the projection of the viewplane of the solid occluder's volume Vol and the squared distance of the occluder from the viewpoint:

$$
f_{\text {solid }}=\frac{V o l}{\|\vec{V}\|} \cdot\|\vec{V}\|^{-2}=\frac{V o l}{\|\vec{V}\|^{3}}
$$

Keep in mind that the optimisation function for the solid occluders does not depend on angular attributes as the near plane of the constructed frustum always faces the centre of projection (eq. (2)). $f_{\text {planar }}$ and $f_{\text {solid }}$ are balanced and do not need further biasing to become compatible.

The effectiveness of the solid occluders becomes apparent when moving among the buildings, especially at ground level and at inspection distance (near). Planar occluders would mostly produce partial occlusion when not facing the main sides of the buildings straight on. Most of the time we view the blocky buildings from odd angles and that is where the solid occluders provide a unified contiguous frustum to take into account all sides at once.

\subsection{Skylight Illumination Model}

Most realistic rendering in VR has dealt with indoor scenes or has focused on objects and buildings. However, outdoor scenes differ from indoor ones in two important aspects, other than geometry: most of their illumination comes directly from the sun and sky; and the distances involved make the effects of air "aerial perspective" visible. A Skylight Illumination Model, once incorporated into the framework, captures 
and simulates the visual aspects and results of these phenomena. It changes sunlight position and colour from the pale red of dawn to the bright yellow of midday and back again. It determines the colour and brightness of the sky throughout the day and it cues us to the distance of objects by shifting their colours. All these effects vary not only based on time of day, but also depending on weather, pollution and other factors.

Traditionally, outdoor VR applications would model these phenomena using Skydomes or Skyboxes, texture-mapped with static high-resolution sky and cloud renderings. In our latest outdoor simulation of ancient Olympia, a sky simulation from sunrise to sunset and nightfall was needed. Therefore, the implementation of a Skylight model was necessary.

The analytic model of Preetham et al [14] was implemented with some modifications regarding the color conversion algorithm to produce more dramatic sunsets/sunrises and the incorporation of a nightfall simulation with rendering of stars and moon (Fig. 3).

An EVS node was created which constructs the dome geometry takes as input the sun position and weather conditions and finally, draws the dome, computing the colours for every vertex with analytic formulas. The colour and intensity of the sun is also computed and all underlying geometry is lit by its computed light attributes. Indirect skylight illumination, caused by the atmospheric scattering of light, is simulated by additional light sources placed in the perimeter of the scene. These light sources are linked to the simulation and their intensity and color is controlled to match their respective position in the skylight simulation.

The initial implementation proposed in [14] only computed values during the day. To overcome this limitation, at nightfall, the sky and sunlight colours get interpolated to a standard night bluish colour. During the night, stars and the moon are faded in, implementing a fast and easy night sky model.

All the weather parameters and the position of the sun can be animated to produce smooth animations of sunset or sunrise along with changes in weather conditions.

\section{Conclusions}

As the curtain of the ATHENS 2004 Olympic Games came down, the odyssey of creating this series came to an end, confirming and outlining once again the importance of combining interactivity, storyboard with culture, advanced graphics and social interest points. All these key factors lead to the success and embracement of this series of applications from the public. Encouraged from the visitor numbers (approx. 7500) during the summer of the Olympiad and their positive feedback, we feel convinced that we succeeded in the challenge of bringing Ancient Olympia to the public and comfortable in undertaking and pushing future projects into new directions.

\section{References}

1. Cal3D, Character Animation Library: http://cal3d.sourceforge.net/

2. Cohen-Or, D., Chrysanthou, Y., Silva, C., Durand, F.: A Survey of Visibility for Walkthrough Applications. IEEE TVG 9, 3 (2003), 412-431.

3. Coldet Collision Detection Library: http://photoneffect.com/coldet/ 
4. Coorg, S., Teller, S.: Real-time occlusion culling for models with large occluders. In Proc. ACM Symposium on Interactive 3D Graphics, 1997, 83-90.

5. Cruz-Neira, C., Sandin, D. J., DeFanti, T. A.: Surround-Screen Projection-Based Virtual Reality: The Design and Implementation of the CAVE. In Proc. ACM Computer Graphics (SIGGRAPH) 1993, 135-142

6. Eberly, D. H.: Game Physics. Morgan Kaufmann, 2004.

7. Gaitatzes, A., Christopoulos, D., Papaioannou, G.: The Ancient Olympic Games: Being Part of the Experience. In Proc. 5th International Symposium on Virtual Reality, Archaeology and Cultural Heritage (VAST) 2004.

8. Gaitatzes, A., Christopoulos, D., Roussou, M.: Reviving the Past: Cultural Heritage Meets Virtual Reality. In Proc. Virtual Reality, Archaeology and Cultural Heritage (VAST) 2001.

9. Gaitatzes, A., Christopoulos, D., Roussou, M.: Virtual Reality Interfaces for the Broad Public. In Proc. Panhellenic Conference on Human Computer Interaction (PCHCI ) 2001.

10. Hudson, T., Manocha, D., Cohen, J., Liny, M., Ho, K., Zhang.: Accelerated Occlusion Culling using Shadow Frusta. In Proc. ACM 13th Annual Symposium on Computational Geometry, 1997, 1-10.

11. Papaioannou, G., Gaitatzes, A., Christopoulos, D.: Enhancing Virtual Walkthroughs of Archaeological Sites. In Proc. 4th International Symposium on Virtual Reality, Archaeology and Cultural Heritage (VAST) 2003.

12. Pape, D., Imai, T., Anstey, J., Roussou, M., DeFanti, T.: XP: An Authoring System for Immersive Art Exhibitions. In Proc. VSMM 1998, Gifu, Japan.

13. Preece, J., Rogers, Y., Sharp, H., Benyon, D., Holland, S., Carey, T.: Human - Computer Interaction. Addison - Wesley, 1994.

14. Preetham, A., Shirley, P., Smits, B. E.: A Practical Analytic Model for Daylight. In Proc. ACM SIGGRAPH 1999, 91-100.

15. Rohlf, J., Helman, J.: IRIS Performer: A High-Performance Multiprocessing Toolkit for Real-Time 3D Graphics. In Proc. ACM SIGGRAPH 1994, 381-395.

16. Taylor, R. M., Hudson, T. C., Seeger, A., Weber, H., Juliano, J., and Helser, A. T.: VRPN: A Device-Independent, Network-Transparent VR Peripheral System. In Proc. ACM Symposium on Virtual Reality Software and Technology (VRST), Banff, Alberta, Canada, November 2001.

17. Watt, A., Watt, M.: Advanced Animation and Rendering Techniques, Theory and Practice. Addison - Wesley, 1993.

18. Woodland, R.: Filling the Gaps - Advanced Animation Using Stitching and Skinning. Game Programming Gems, Ed. M. DeLoura, 2000, Charles River Media, 476-483. 